\title{
Determination of load-transfer parameters of single piles axially loaded
}

\section{Détermination des paramètres de transfert de charges d'un pieu isolé chargé axialement}

\author{
Yaich Achour $\mathrm{N}^{1}$, Bouafia $\mathrm{A}^{2}$ \\ ${ }^{1}$ Ecole Nationale Supérieure de l'Hydraulique (ENSH), Blida, Algérie \\ ${ }^{2}$ Université de Saad Dahleb, Blida, Algérie
}

\begin{abstract}
Modern buildings require more use of the deep foundations including piles. The good perception of the behavior of piles and the knowledge of their bearing capacity is essential for a rational use. The present work focuses on the behavior of single pile under vertical loads in clay and sand soils. Thus, an approach to semi-empirical calculations load transfer as well as finite element modeling are used in order to determine the curve loading / sag at the head of pile and deduce the calculation parameters. This study has allowed predicting the load curves piles, knowing their geometric properties and some test results of in-situ soil (CPT, PMT and SPT).
\end{abstract}

\section{Introduction}

Très souvent, les couches d'un terrain sont compressibles, tandis que les couches sous-jacentes, à partir d'une certaine profondeur, sont de meilleure résistance. Autrefois, la plupart des bâtiments étaient construits sur des fondations superficielles et lorsque le sol de surface était compressible, on battait un nombre important de pieux en bois. A partir du $18^{\text {ème }}$ siècle, les immeubles deviennent de plus en plus lourds et rigides et les ingénieurs ont cherché avec plus de précision quelle était la portance réelle des pieux, les tassements maximums et les fiches minimales nécessaires. L'étude de ces paramètres par l'essai de chargement expérimental est très coûteuse et, qui est d'ailleurs, réservée pour les grands projets. Ces derniers temps, pour résoudre ce problème, beaucoup de recherches sont conduites afin d'aboutir à des méthodes de calcul des pieux, en déterminant leurs capacité portante et leurs déformations en fonction des caractéristiques du sol autour du pieu, déterminées à partir d'essais in-situ, telle que CPT,PMT et le SPT $[1,2]$.

Le présent travail vise à étudier le comportement d'un pieu isolé chargé axialement, dans l'objectif de déterminer la courbe charge/enfoncement en tête du pieu en utilisant une approche de calcul basée sur une méthode semi-empirique de transfert de charge. A fin de déterminer les paramètres de transfert nécessaires dans la dite méthodologie, une modélisation par éléments finis du pieu est essentielle.

Par ailleurs les courbes de chargement sont déterminées à l'aide du programme de calcul SETPIL
(Settelment of Piles), voué au calcul des pieux isolés soumis à des sollicitations verticales. Dans cette étape, les d'essais in-situ (CPT, PMT et SPT) sont exploités. Finalement, ces courbes seront comparées aux courbes des essais de chargement réel et des expressions pour les paramètres de calcul seront proposées.

\section{Détermination des paramètres de transfert de charge $B_{0}$ et $R_{0}$}

\subsection{Méthodologie}

La portance d'un pieu est le cumul de la résistance en pointe $\mathrm{q}_{1}$ et celle du frottement latéral de l'interface sol/pieu. Dans le domaine des faibles tassements, la pression verticale en pointe est caractérisée par un paramètre de déformation $\mathrm{R}_{0}$, et des contraintes de frottement latéral $(\tau)$ qui sont caractérisées par un paramètre de mobilisation de frottement $\mathrm{B}_{0}$. Ces deux paramètres sont utilisés dans la théorie de transfert de charges pour évaluer les valeurs des tassements du pieu chargé verticalement. On suppose que les contraintes mobilisées à l'interface sol/pieu, à une profondeur (z) donnée, sont proportionnelles au tassement correspondant (v) par les équations 1 et $2[3,4]$ :

$$
\begin{aligned}
& \tau(z)=B_{0}(z) \cdot v(z) \\
& q_{p}=R_{0} \cdot \frac{v(D)}{B}
\end{aligned}
$$

Avec : D : Fiche, B : Diamètre du pieu. 
Dans cette partie d'étude, afin de déterminer les paramètres $B_{0}$ et $R_{0}$, une étude paramétrique a été conduite, en faisant varier les valeurs de compressibilité $\mathrm{K}$ et d'élancement du pieu $\mathrm{D} / \mathrm{B}$ et cela pour un sol homogène ainsi qu'un sol de Gibson. Le calcul des contraintes étant réalisé par une modélisation de l'ensemble pieu/sol par éléments finis, en utilisant le logiciel SAP. Les paramètres $\mathrm{B}_{0}$ et $\mathrm{R}_{0}$ finaux sont déterminés par un ajustement hyperbolique par moindres carrés des courbes de variation obtenues. Dans ce cas, des éléments plans à comportement linéaire sont utilisés.

\subsection{Définition du modèle axisymétrique pieu/sol}

Le problème a été modélisé par un système axisymétrique dont l'axe de symétrie est celui du pieu. Cela traduit le fait que les points diamétralement opposés, subissent les mêmes états de contraintes et de déformations. Donc en tenant compte de la symétrie axiale du problème, uniquement un demi-plan du modèle à été étudié et calculé comme l'illustre la figure 1 .

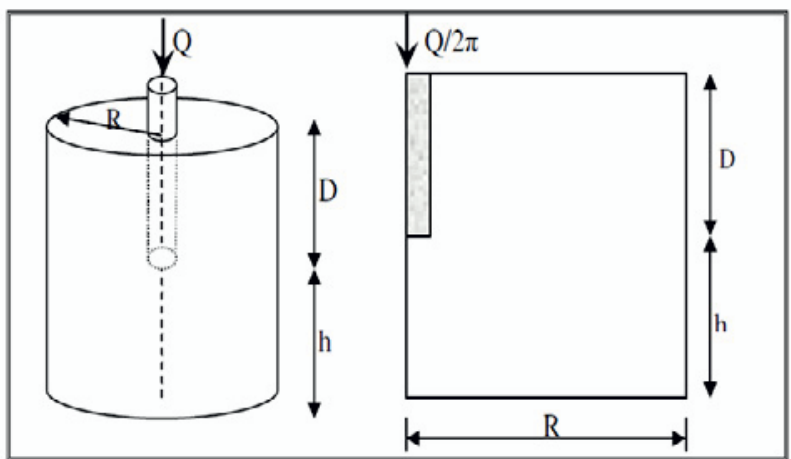

Fig. 1. Courbe de stabilisation des tassements en tête du pieu

Les limites verticales et horizontales du maillage [5] ont été optimisées pour permettre la stabilité des déplacements des points du pieu. A titre d'exemple, la figure 2 présente la variation du déplacement en tête du pieu avec la dimension verticale du maillage. De ce fait, dans le modèle étudié, les limites des maillages utilisés ont été optimisées à $R \approx 25.5 B$ horizontalement et $(D+h)$ verticalement, avec h égale à 2 fois la fiche du pieu $\mathrm{D}$.

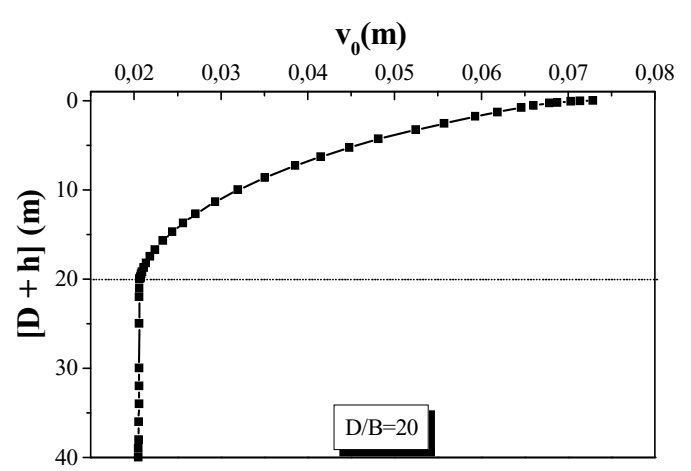

Fig. 2. Courbe de stabilisation des tassements en tête du pieu

\subsection{Résultats et interprétation}

Notons que l'effet de la variation du coefficient de Poisson $v_{\mathrm{s}}$ à court et à long terme, étudiée ultérieurement [6] reste faible. A cet effet, dans cette partie d'étude, la variation de $R_{0} / E_{s}$ et de $B . B_{0} / E_{s}$ en fonction de $D / B$ pour différentes compressibilités $\mathrm{K}$, pour un sol homogène et un sol de Gibson sera réalisée seulement au long terme. Ces variations sont présentées dans les figure 3 et 4 respectivement pour $\mathrm{R}_{0} / \mathrm{E}_{\mathrm{s}}$ et B. $\mathrm{B}_{0} / \mathrm{E}_{\mathrm{s}}$.
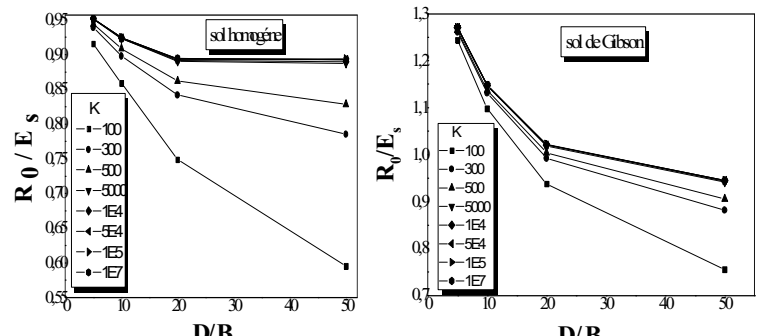

Fig. 3. Variation du rapport $\mathrm{R}_{0} / \mathrm{E}_{\mathrm{s}}$ pour un sol homogène et un sol de Gibson
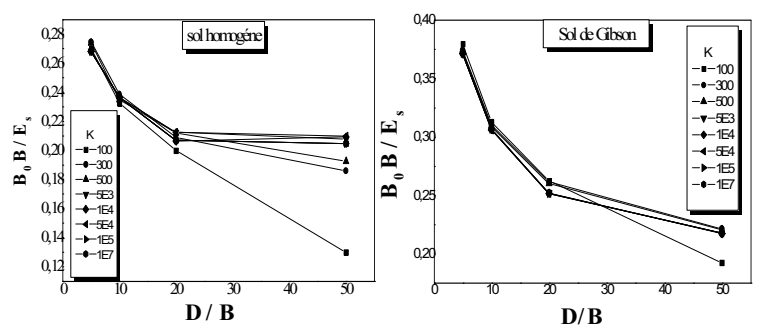

Fig. 4. Courbes B. $B_{0} /$ Es pour un sol homogène et un sol de Gibson

D'après cette analyse, on observe que les paramètres adimensionnels $\mathrm{R}_{0} / \mathrm{E}_{\mathrm{s}}$, et $\mathrm{B}_{0} \mathrm{~B} / \mathrm{E}_{\mathrm{s}}$ diminuent avec l'élancement $\mathrm{D} / \mathrm{B}$ quelque soient le coefficient $\mathrm{K}$ et le type du sol. Pour un élancement relatif $\mathrm{D} / \mathrm{B}$ fixé, le paramètre $\mathrm{R}_{0} / \mathrm{E}_{\mathrm{s}}$ augmente avec la compressibilité relative tant que cette dernière reste inférieure à 5000 , et au delà de cette valeur, ce rapport varie peu avec la compressibilité et cela pour un sol homogène et de Gibson.

Pour le paramètre de frottement, l'effet de la compressibilité relative $\mathrm{K}$ reste négligeable. La figure 4 montre bien que les courbes B. $\mathrm{B}_{0} / \mathrm{E}_{\mathrm{s}}$ de différentes compressibilité sont presque superposés. Une légère influence est à remarquer pour une compressibilité inférieure à 300 et pour un élancement relatif au-delà de 20.

L'ajustement hyperbolique des valeurs obtenues de $\mathrm{R}_{0} / \mathrm{E}_{\mathrm{s}}$ et $\mathrm{B}_{0} \mathrm{~B} / \mathrm{E}_{\mathrm{s}}$ en fonction de $\mathrm{K}$ et $\mathrm{D} / \mathrm{B}$, est représenté par les équations 3 et 4 pour un sol homogène et 5 et 6 pour un sol de Gibson, avec des coefficients de corrélations supérieurs a 0.93 .

$$
\begin{aligned}
& \frac{R_{0}}{E_{s}}=1.04\left(\frac{D}{B}\right)^{-\left[0.03+\frac{15}{K}\right]} \\
& \frac{B B_{0}}{E_{S}}=0.34\left(\frac{D}{B}\right)^{-\left[\frac{0.13 K}{K+56}\right]}
\end{aligned}
$$




$$
\begin{aligned}
& \frac{R_{0}}{E_{S}}=1.61\left(\frac{D}{B}\right)^{-\left[0.13+\frac{8}{K}\right]} \\
& \frac{B B_{0}}{E_{S}}=\left[0.32+\frac{27}{K}\right]\left(\frac{D}{B}\right)^{-\left[0.14+\frac{15}{K}\right]}
\end{aligned}
$$

\subsection{Validation du modèle}

Pour vérifier les formulations de $\mathrm{B}_{0}$ et $\mathrm{R}_{0}$ obtenues par le modèle d'éléments finis, on a fait une comparaison entre les tassements en tête du pieu calculés par la formule de transfert de charge, et les tassements obtenus par la modélisation par éléments finis (logiciel SAP). L'équation (7) [7] présentée ci-après, représente la formule du tassement par la méthode de transfert de charge, les paramètres $\mathrm{B}_{0}$ et $\mathrm{R}_{0}$ sont remplacés par ceux trouvés dans cette étude pour un sol homogène et de Gibson à long terme.

$$
\begin{aligned}
& \mathrm{v}_{0}=\frac{4 \cdot Q}{\pi \cdot B} \frac{1+\frac{R_{0}}{a \cdot B \cdot E_{p}} \operatorname{Tanh}(a \cdot D)}{R_{0}+a \cdot B \cdot E_{p} \cdot \operatorname{Tanh}(a \cdot D)} \\
& \text { Avec } a=\sqrt{\frac{4 \cdot B_{0}}{E_{p} \cdot B}}
\end{aligned}
$$

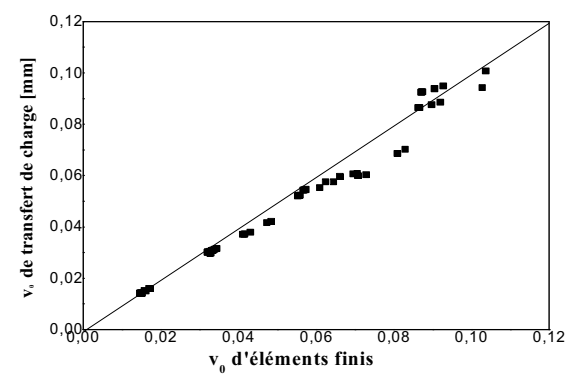

fig. 5. Comparaison entre les Tassements calculés et les tassements d'éléments finis

A partir de la figure 5, on peut déduire que les formulations proposées de $\mathrm{B}_{0}$ et $\mathrm{R}_{0}$ permettent de bien décrire les résultats obtenus par une modélisation par éléments finis. On peut ainsi, pour les besoins de calcul manuel, utiliser les formulations ci-dessus (Equations 3$6)$.

\section{Détermination des courbes de chargement}

\subsection{Méthodologie de calcul}

Dans cette partie, les courbes de chargement sont déterminées, en fonction des paramètres de transfert $\mathrm{B}_{0}$ et $\mathrm{R}_{0}$ déduits précédemment ainsi que les contraintes de frottement $\mathrm{q}_{\mathrm{s}}$ et en pointe $\mathrm{q}_{\mathrm{L}}$ (déterminées à partir des essais in-situ), moyennant le programme de calcul SETPIL [8]. Ce dernier se base sur une méthode d'analyse non linéaire de calcul des pieux isolés chargés verticalement utilisant la théorie de transfert de charge, afin de simuler l'interaction sol/pieu. La méthodologie utilisée par le programme SETPIL est bien détaillée par Bouafia [8].

La base de données expérimentales utilisée est construite à partir d'une collecte de données de la littérature concernant des pieux chargés verticalement, essentiellement circulaires en béton, mis en place dans des sols argileux ou sableux issus de plusieurs sites (Belgique, Canada, Italie,...) [9].

Les données principales exploitées sont les résultats des essais CPT, PMT et SPT ainsi que les courbes de chargement/enfoncement réelles des pieux.

La détermination des valeurs de la pression limite du frottement latéral $\mathrm{q}_{\mathrm{s}}$ et celle de la contrainte limite en pointe du pieu $q_{p}$ se fait en fonction de la résistance pénétrométrique $\left(\mathrm{q}_{\mathrm{c}}\right)$, du module pressiométrique $\left(\mathrm{E}_{\mathrm{m}}\right)$ et la pression limite $\left(\mathrm{P}_{1}\right)\left[10\right.$ ] et le nombre de battage $\left(\mathrm{N}_{\mathrm{SPT}}\right)$ à partir des corrélations avec les essais in-situ [11] :

- Essais CPT : $\mathrm{q}_{\mathrm{s}}=f\left(\mathrm{q}_{\mathrm{c}}\right)$ et $\mathrm{q}_{\mathrm{p}}=f\left(\mathrm{q}_{\mathrm{c}}\right)$,

- $\quad$ Essais PMT : $\mathrm{q}_{\mathrm{s}}=f\left(\mathrm{E}_{\mathrm{m}}, \mathrm{P}_{\mathrm{l}}\right)$ et $\mathrm{q}_{\mathrm{p}}=f\left(\mathrm{E}_{\mathrm{m}}, \mathrm{P}_{1}\right)$,

- $\quad$ Essais SPT : $\mathrm{q}_{\mathrm{s}}=f\left(\mathrm{~N}_{\mathrm{SPT}}\right)$ et $\mathrm{q}_{\mathrm{p}}=f\left(\mathrm{~N}_{\mathrm{SPT}}\right)$.

Les paramètres sont introduits dans un fichier donné du programme SETPIL. Le résultat obtenu, représente la courbe de chargement-tassement en tête du pieu $\left(\mathrm{Q}-\mathrm{v}_{0}\right)$. Cette courbe sera comparée avec la courbe du chargement réelle. Cette comparaison est évaluée en calculant un coefficient de corrélation R. Dans le cas où la corrélation est faible $(<0,8)$, le calcul est refait en introduisant un coefficient correcteur des paramètres calculés auparavant. En fin, des expressions des paramètres de calcul sont obtenues en regroupant les valeurs des paramètres de transfert de charge $B_{0}$ et $R_{0}$ ainsi $\mathrm{q}_{1}$ et $\mathrm{q}_{\mathrm{s}}$ qui ont conduit à de bons résultats (Facteur de corrélation $\mathrm{R}>0.8$ ).

\subsection{Présentation des résultats}

A fin d'expliciter les étapes d'étude, on présente ciaprès un exemple de calcul d'un cas d'essai réelle de chargement d'un pieu dans le site à Garigliano (Italie). Le pieu a un diamètre de $0.381 \mathrm{~m}$ et une longueur de $48 \mathrm{~m}$, et foré dans une couche d'argile molle.

Après un calcul des paramètres d'entrée $\left(B_{0}, R_{0}, q_{1}\right.$, $\mathrm{q}_{\mathrm{s}}$ ), et une compilation du programme SETPIL, la courbe de chargement $\left(\mathrm{Q}-\mathrm{v}_{0}\right)$ simulée obtenue est comparée avec la courbe $\left(\mathrm{Q}-\mathrm{v}_{0}\right)$ expérimentale. Ainsi, le facteur de corrélation $\mathrm{R}$ obtenu entre les deux courbes est de 0.7 , montrant relatives divergences entre les deux courbes de chargement.

A fin d'y remédier, on fait appel à des coefficients correcteurs pour les paramètres d'entrée initiaux et des compilations successives du programme SETPIL ont été réaliser jusqu'à obtention de la bonne courbe de chargement conduisant à une bonne corrélation, correspondante à $\mathrm{R}>0.8$. Ainsi, après ce traitement, comme le montre la figure 6 , une bonne corrélation $(\mathrm{R}=0.92)$ entre les deux courbes de chargement a été obtenue. En se basant sur le même principe, la figure 7 présente quelques exemples de l'analyse des essais de chargement vertical d'autres pieux issus de la base de données. 


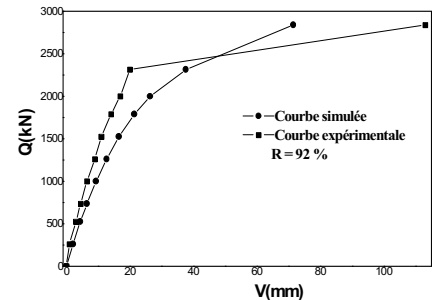

fig. 6. Courbe de chargement expérimental et de SETPIL, site de Garigliano (Italie)
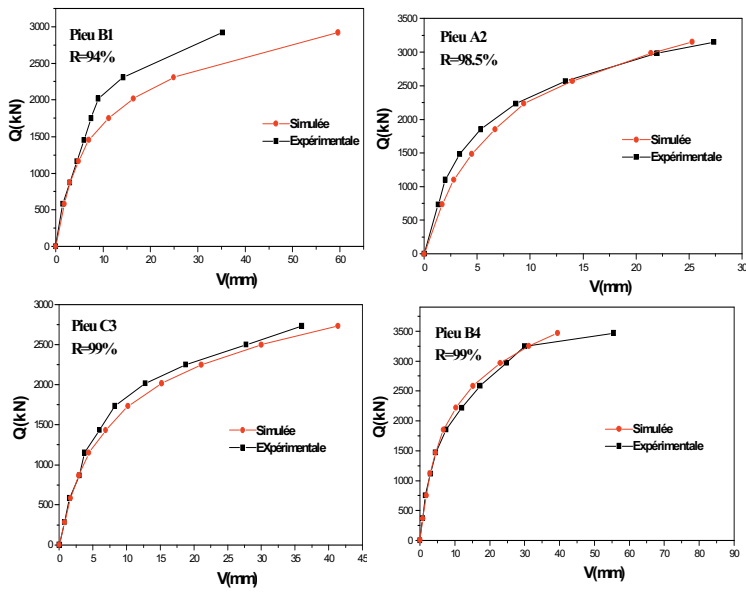

fig. 7. Comparaison des courbes de chargement- sols sableux site Limlette (Belgique)

Les expressions des paramètres de calcul ont été obtenues en regroupant les valeurs des paramètres de transfert de charge $\mathrm{B}_{0}$ et $\mathrm{R}_{0}$ ainsi $\mathrm{q}_{1}$ et $\mathrm{q}_{\mathrm{s}}$ corrigés. Faisant le calcul de moyenne de ces derniers ainsi que l'écart type et le coefficient de variation pour ces valeurs, on obtient les résultats présentés ci-après :

\section{$\checkmark \quad$ Cas du CPT}

- La pente initiale $B_{0}$ de la courbe $(\tau-v)$, est obtenue en calculant la moyenne, et on adopte l'équation 9 pour le calcul de $\mathrm{B}_{0}$, pour le cas des sols argileux et sableux.

$\mathrm{B}_{0}=2.5 \frac{q_{c}(z)}{B} \quad($ en $\mathrm{kPa} / \mathrm{m})$

- On se propose pour le calcul de la pente initiale $\mathrm{R}_{0} \mathrm{de}$ la courbe $\left(\mathrm{q}_{\mathrm{p}}-\mathrm{v} / \mathrm{B}\right)$, pour le cas des sols argileux et sableux, l'équation 10 suivante :

$\mathrm{R}_{0}=15 \times \mathrm{q}_{\mathrm{c}}(\mathrm{D}) \quad($ en $\mathrm{kPa})$

- La détermination de la résistance limite de frottement latéral se fait par le biais des deux équations 11 et 12 et cela pour les argiles et les sables respectivement, et celle de la pointe du pieu est donné par l'équation 13 pour les argiles et les sables :
$\mathrm{q}_{\mathrm{s}}=3 \mathrm{q}_{\mathrm{s}}^{\max }$
(en $\mathrm{kPa})$
$\mathrm{q}_{\mathrm{s}}=\mathrm{q}_{\mathrm{c}} / 57$
(en $\mathrm{kPa})$
$\mathrm{q}_{1}=\mathrm{K}_{\mathrm{c}} \cdot \mathrm{q}_{\mathrm{c}}{ }^{\mathrm{e}}$
(en $\mathrm{kPa})$

Les valeurs de $\mathrm{K}_{\mathrm{c}}$, et $\mathrm{q}_{\mathrm{s}}^{\max }$ sont regroupées dans le tableau1.
Tableau 1. Choix des $\mathrm{K}_{\mathrm{c}}$ et $\mathrm{q}_{\mathrm{s}}{ }^{\max }$

\begin{tabular}{|c|c|c|c|c|c|}
\cline { 3 - 6 } \multicolumn{2}{c|}{} & \multicolumn{2}{c|}{ Pieu battu } & \multicolumn{2}{c|}{ Pieu foré } \\
\hline \multirow{2}{*}{$\mathrm{K}_{\mathrm{c}}$} & Argile & \multicolumn{2}{|c|}{1.10} & \multicolumn{2}{c|}{0.40} \\
\cline { 2 - 6 } & Sable & \multicolumn{2}{c|}{0.2} & \multicolumn{2}{c|}{0.5} \\
\hline \multirow{4}{*}{$\mathrm{q}_{\mathrm{s}}{ }^{\text {max }}(\mathrm{kPa})$} & Argile molle & 15 & Argile molle & 15 \\
\cline { 2 - 6 } & Argile Ferme & 40 & Argile Ferme & 40 \\
\cline { 2 - 6 } & $\begin{array}{c}\text { Argile très } \\
\text { ferme à dure }\end{array}$ & 80 & $\begin{array}{c}\text { Argile très } \\
\text { ferme à dure }\end{array}$ & 40 \\
\hline
\end{tabular}

\section{$\checkmark$ Cas du PMT}

Dans le cas du pressiométre, les paramètres nécessaires pour la détermination de la courbe de chargement des pieux sont comme suit :

$$
\begin{aligned}
& \mathrm{B}_{0}=2 \frac{E_{m}(z)}{B} \quad(\text { en } \mathrm{kPa} / \mathrm{m}) \\
& \mathrm{R}_{0}=10^{*} \mathrm{E}_{\mathrm{m}}(\mathrm{D}) \quad(\text { en } \mathrm{kPa}) \\
& \mathrm{q}_{1}=2 \mathrm{p}_{\mathrm{le}}^{*} \quad(\text { en } \mathrm{kPa})
\end{aligned}
$$

Le calcul de la contrainte de frottement latéral se fait par le biais des deux courbes $\mathrm{Q}_{1}$ et $\mathrm{Q}_{2}$, pour le cas des pieux en béton foré et battus respectivement dans les argiles, et la courbe $\mathrm{Q}_{3}$ pour les pieux vissés dans le sable.

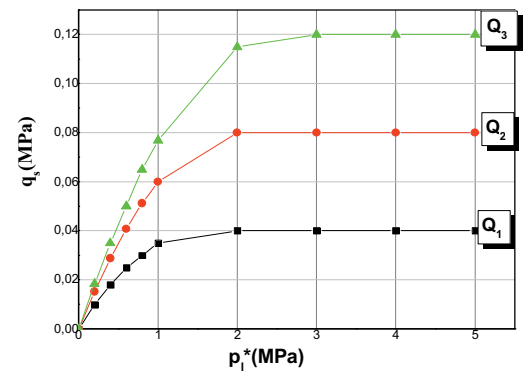

Figure .8. Frottement latéral $\mathrm{q}_{\mathrm{s}}$ en fonction de $\mathrm{p}_{1}{ }^{*}$

\section{$\checkmark \quad$ Cas du SPT}

Dans le pénétromètre standard, les paramètres de calcul sont exprimés en fonction de nombre de battage $\mathrm{N}$, comme le montre les équations ci-après :

$$
\begin{aligned}
B_{0} & =4023 \frac{N^{c o r}(z)}{B} \quad(\mathrm{kPa} / \mathrm{m}) \\
\mathrm{R}_{0} & =17550 * \mathrm{~N}^{\mathrm{cor}}(\mathrm{D}) \quad(\mathrm{kPa}) \\
\mathrm{q}_{\mathrm{s}} & =4.14 * N_{\text {moy }}^{c o r} \\
\mathrm{q}_{1} & =120 * N_{\text {moy }}^{c o r}(\mathrm{D}-3 \mathrm{~B}, \mathrm{D}+3 \mathrm{~B})
\end{aligned}
$$

Avec

$\mathrm{N}^{\text {cor }}$ : c'est la valeur corrigée du nombre de coups $\mathrm{N}$ par l'effet de la nappe, ayant des valeurs de $\mathrm{N}$ plus grandes que 15, la formule donnée par Terzagui est comme suit :

$\mathrm{N}^{\text {cor }}=15+\frac{N-15}{2}$ 
$N_{\text {moy }}^{\text {cor }}$ : Moyenne arithmétique des valeurs du nombre $\mathrm{N}$ mesuré le long du pieu.

$N_{\text {moy }}^{c o r}$ (D-8B,D+3B): c'est la moyenne arithmétique des valeurs du nombre de coups $\mathrm{N}$ dans la zone utile de résistance en pointe définie à 8 fois le diamètre au-dessus de la pointe et 3 fois le diamètre au-dessous.

\subsubsection{Validation des expressions}

Afin de tester la validité de nos expressions, une comparaison a été établie pour les tassements en tête du pieu calculés et expérimentales (Base de données : Essais in-situ CPT, PMT et SPT). La figure 9 montre une bonne corrélation entre les tassements réels et ceux calculés par la méthodologie développée, notamment pour les sols sableux.

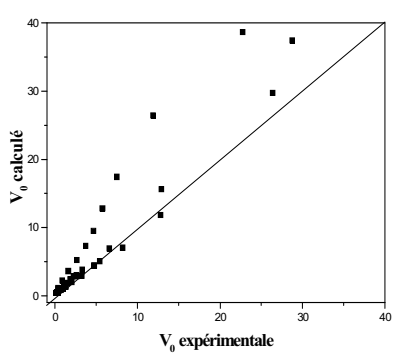

a- Sols Argileux

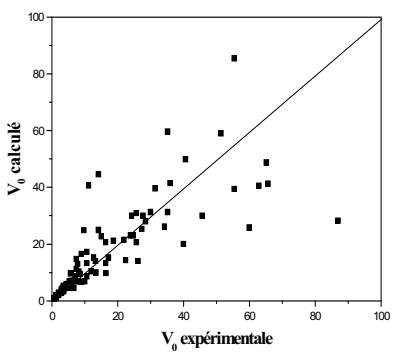

b- Sols Sableux
Fig. 9. Comparaison entre les tassements de calculs et réels

\section{Conclusion}

La capacité portante des pieux isolés peut être déterminée à l'aide des méthodes classiques de la mécanique des sols, et aussi par des méthodes de calcul ce basant sur des essais in-situ. En outre, la capacité portante peut être évaluée à partir des essais de chargement sur pieu en vraie grandeur, qui reste est un moyen très coûteux.

Ce travail, qui s'est intéressé principalement à des pieux isolés circulaires en béton chargés axialement dans des sols sableux ou argileux, nous a permis de mettre en évidence des formulations pour les paramètres de transfert de charge ainsi que les contraintes de frottement et en pointe du pieu. Ces paramètres, qui sont en fonction des caractéristiques du sol déterminées à partir des essais in-situ (CPT, PMT, SPT), permettent de simuler les courbes de chargement des pieux, qui corrèlent bien avec les courbes de chargement réelles.

Cette méthodologie, qui a fait intervenir la théorie de transfert de charge ainsi que la modélisation par éléments finis, peut être élargie pour d'autre type de pieux et avec des sols multicouches.

\section{References}

1. A. Eslami, and H. F. Bengt, Bearing Capacity of Pile from Cone Penetration Test (CPT) Data, International Symposium on Cone Penetration Testing, CPT'95, 2, 453-460, (1995)
2. M. Roy, L. Tanguay, Capacité portante des pieux isolés dans les argiles sensibles: étude de cas par la méthode pénétromètrique, Canadian Geotechnical Journal, 26, 375-383, (1989)

3. A. Bouafia, Les essais in-situ dans les projets de fondations, OPU, Alger, (2004)

4. R. Frank, Calcul des Fondations Superficielles et Profondes, Techniques de l'Ingénieur et presse de l'ENPC, (1999).

5. Ph. Mestat, Maillages d'éléments finis pour les ouvrages de géotechnique - Conseils et recommandations, Bull. Labo $\mathrm{P}$ et $\mathrm{Ch} . \mathrm{N}^{\circ} 212$, 39-64, (1997)

6. N. Yaich Achour, A. Bouafia, A. Hadj-Sadok, Détermination de la courbe de chargement d'un pieu isolé chargé axialement, 1er Congrès international de génie civil et d'hydraulique Guelma, Algerie (2012).

7. A. Bouafia, Mécanique des sols appliquée, OPU, Alger, (2000)

8. A. Bouafia, Manuel de SETPIL: Calcul non linéaire des pieux chargés axialement- Manuel d'utilisation, (1996).

9. N. Yaich Achour, Paramètre de transfert de charge des fondations profondes; Analyse d'une base de donnée, Thèse de magister, département de génie civil, Université de Saad Dahleb, Blida, Algerie (2004)

10. M. Bustamante, R. Frank, et S. Christoulas, Revue française de géotechnique, 39, 54 (1991)

11. M. Cassan, Les essais in situ en mécanique des sols, Tome II : Applications et Méthodes de Calcul, Edition Eyrolles, (1978) 